\title{
Role of tyrosine phosphorylation in the antioxidant effects of the p75 neurotrophin receptor
}

\author{
Tong Zhang, Zhiping Mi and Nina F. Schor*
}

Department of Pediatrics and the Center for Neural Development and Disease; University of Rochester Medical Center; Rochester, NY USA

Key words: neurotrophins, neurotrophin receptors, reactive oxygen species, oxidative stress, apoptosis, tyrosine phosphorylation

The p75 neurotrophin receptor (p75NTR) is an $\alpha$ - and $\gamma$-secretase substrate expressed preferentially in the cholinergic neurons of the nucleus basalis of Meynert, the hippocampus, and the cerebellum of the adult brain. Mutations of the $\gamma$-secretase, presenilin, have been implicated in familial Alzheimer's disease. Furthermore, oxidative and inflammatory injury to the cholinergic neurons of the nucleus basalis of Meynert and hippocampus plays a critical role in the pathology of Alzheimer's disease. The intracellular domain of p75NTR (p75ICD) is the $\alpha$ - and $\gamma$-secretase cleavage fragment of the holoreceptor that functions as an antioxidant in PC12 rat pheochromocytoma cells. Phosphorylation of the receptor is thought to be necessary for many of its functions, and two tyrosines in p75ICD have been among the functionally important phosphorylation sites. Site-directed mutagenesis was used to generate three p75ICD mutants that cannot be phosphorylated at either or both tyrosines, respectively. Each of these mutants was expressed in p75NTR-deficient PC12 cells to determine the effects of blocking phosphorylation at specific sites on the antioxidant activity of p75ICD. Interfering with phosphorylation at tyrosine-337 impairs antioxidant function, while interfering with phosphorylation at tyrosine-366 does not, and may in fact impart protection from oxidant stress. Neither MAPK (i.e., p38, ERK1, ERK2) content nor NF- $\mathrm{B}$ activation accounts for the differential sensitivity to oxidant stress among the differentially phosphorylated p75NTR cell lines. However, differences in the time course of ERK1,2 phosphorylation among the lines account in large measure for their differential oxidant sensitivity. The phosphorylation state of specific sites on p75ICD may modulate the resistance of neurons in Alzheimer's disease-relevant brain regions to oxidant stress.

${ }^{*}$ Correspondence to: Nina F. Schor; Department of Pediatrics; Golisano Children's Hospital; University of Rochester Medical Center; 601 Elmwood Avenue; Box 777; Rochester, NY 14642 USA; Tel.: 585.275.4673; Fax: 585-273-1079; Email: nina_schor@urmc.rochester.edu

Submitted: 08/04/09; Revised: 08/05/09; Accepted: 08/06/09

Previously published online as an Oxidative Medicine and Cellular Longevity E-publication:

www.landesbioscience.com/journals/oximed/article/9745

\section{Introduction}

The p75 neurotrophin receptor (p75NTR) has been shown to mediate antioxidant function in PC12 rat pheochromocytoma cells. ${ }^{1}$ Physiological signaling through p75NTR includes ligand binding and receptor dimerization, cleavage of p75NTR by $\alpha$ and $\gamma$-secretases, ${ }^{2}$ phosphorylation of the intracellular domain (p75ICD) thereby liberated, binding of interactor proteins to phosphorylated p75ICD (with phosphorylation site dependence of the specific interactor that binds), and several interactor-dependent signaling pathways that determine whether p75NTR signaling leads to the life or death of the cell. In circumstances in which "life" signaling is enacted, p75ICD is as effective an antioxidant as full-length p75NTR. ${ }^{1}$ Thus, under physiological circumstances, the nature of the activity of p75NTR depends critically upon its phosphorylation; that is, the specific function of p75NTR in a given milieu is, in part, dictated by the site(s) at which phosphorylation occurs. 3,4

The importance of cleavage of p75NTR, liberation of p75ICD, and the antioxidant signaling this triggers is underscored by the effects of mutation of the $\gamma$-secretase, presenilin-1, in familial Alzheimer's disease. While p75NTR is ubiquitously expressed in embryonic brain, its expression is successively more and more restricted as the fetus matures. In the adult central nervous system, p75NTR is preferentially expressed in the cholinergic neurons of the nucleus basalis of Meynert, the hippocampus, and the cerebellum, loci implicated in the pathology of Alzheimer's disease. Unlike its wildtype counterpart, familial Alzheimer's disease mutant presenilin-1 is unable to cleave p75NTR and p75ICD is not liberated. It is hypothesized that p75NTR antioxidant activity is therefore not present in the neurons of patients with familial Alzheimer's disease. Consistent with this hypothesis, reactive oxygen species have been hypothesized to play a role in the loss of cholinergic neurons in the brains of patients with Alzheimer's disease. ${ }^{1,5,6}$

Coordinate regulation of phosphorylation occurs between Y366 and Y337. Under baseline conditions, blocking phosphorylation by replacing Y366 with F results in increased spontaneous phosphorylation of Y337, and vice versa. However, neither monophosphorylated molecule is as heavily phosphorylated after NGF treatment as wildtype p75NTR. ${ }^{3}$ Furthermore, phosphorylation at 
Y337 is necessary (although not sufficient) for c-Cbl-dependent ubiquitination and consequent internalization and degradation of p75NTR. ${ }^{4}$ In addition, p75NTR-mediated inactivation of the inhibitor of neurite outgrowth, Rho, requires phosphorylation of Y366 within the death domain of p75NTR. This residue lies between helices 2 and 3 and it is hypothesized that phosphorylation at his locus controls spacing between helices 2 and 3 and helices 4 and 5. ${ }^{3}$ Thus, phosphorylation of $\mathrm{p} 75 \mathrm{NTR}$ is one of the arbiters of the function of this receptor.

We sought to examine the effects of phosphorylation of Y337 and Y366 on the antioxidant effects of p75ICD in PC12 cells. Using site-directed mutagenesis, we transfected p75NTR-negative PC12 cells ${ }^{1,7}$ with wildtype and each of three mutant (Y337F, Y366F, and Y337F/ Y366F, respectively) p75ICD expression constructs. Interfering with phosphorylation at Y337 impairs antioxidant function, while interfering with phosphorylation at Y366 enhances neuroprotection. These findings suggest potential therapeutic targets for enhancing (as one might want to do in combating neural tumors) or diminishing (as one might want to do in preventing Alzheimer's disease) the cytotoxic effects of reactive oxygen species in the nervous system.

\section{Results}

Expression and phosphorylation of p75ICD in transfected p75NTR-deficient cells. Western blot analysis demonstrates that p75ICD is expressed in all of the transfectants except the mock transfectants (Fig. 1A and B). Although the relative intensity of the bands varied from blot to blot, on repeated assessment, the p75ICD protein content ( $\mathrm{n}=3$ independent blots) does not differ significantly from transfectant to transfectant ( $p>0.05$ for all paired comparisons; Student's t-test, Bonferroni correction). Maintenance of p75ICD-transfected PC12 cells in the presence of the tyrosine phosphorylase inhibitor, sodium orthovanadate results in detection of phosphorylated $\mathrm{Y}(\mathrm{pY})$ in each of the immunoprecipitated p75ICD mutants (Fig. 1C).

Cell survival after treatment with 6-OHDA. As we have previously reported, ${ }^{1}$ transfection of p75NTR-deficient PC12 cells with wildtype $\mathrm{p} 75 \mathrm{ICD}$ protects them from 6-OHDA-induced cell death (Fig. 2A; p < 0.04, Mann-Whitney $U$ test, aggregate of all points for wild type p75ICD vs. aggregate of all points for mock transfectants). Cells transfected with the Y337F mutant or Y337F/Y366F double mutant, respectively, are more sensitive to 6-OHDA than are wildtype or Y366F mutant transfectants (Fig. 2B; $\mathrm{p}<0.05$, Mann-Whitney $U$ test, aggregate of all points for $\mathrm{Y337F}+\mathrm{Y337F} / \mathrm{Y366F}$ vs. aggregate of all points for wild type + Y366F transfectants). Cells transfected with the Y366F mutant are less susceptible to 6-OHDA-induced cell death than are $\mathrm{Y} 337 \mathrm{~F}$ transfectants $(\mathrm{p}<0.03$, Mann-Whitney $\mathrm{U}$ test, all points for Y366F vs. all points for Y337F transfectants). Double mutants behave more like Y337F than like Y366F mutants. Results obtained using the Alamar blue assay were confirmed using $\mathrm{LDH}$ release into the medium (Fig. 2C).

Assessment of nuclear morphology (20-24 h; Fig. 3A and B) and caspase- 3 cleavage (0 and $6 \mathrm{~h}$; Fig. 4) after 6-OHDA $(200 \mu \mathrm{M})$ treatment suggests the apoptotic nature of 6-OHDAinduced cell death in this model and confirms the importance of phosphorylation of $\mathrm{Y} 337$ for the anti-oxidant protective effects of p75ICD. Interestingly, fractional poly-ADP-ribose polymerase (PARP) cleavage at 6 and $9 \mathrm{~h}$ after addition of 6-OHDA to the medium of mutant p75ICD transfectants (Fig. 5) did not differ significantly from that of wild type p75ICD transfectants, except in the case of the $9 \mathrm{~h}$ determination for the double mutant ( $\mathrm{p}<0.01$ compared to wild type, Student's t-test). Similarly, by $6 \mathrm{~h}$ after treatment with 6-OHDA, the PARP cleavage product is most abundant in double mutant-transfected cells.

Activation of signal transductants by 6-OHDA. Cellular content of activated p38 and ERK1,2, MAP kinases (MAPKs) 


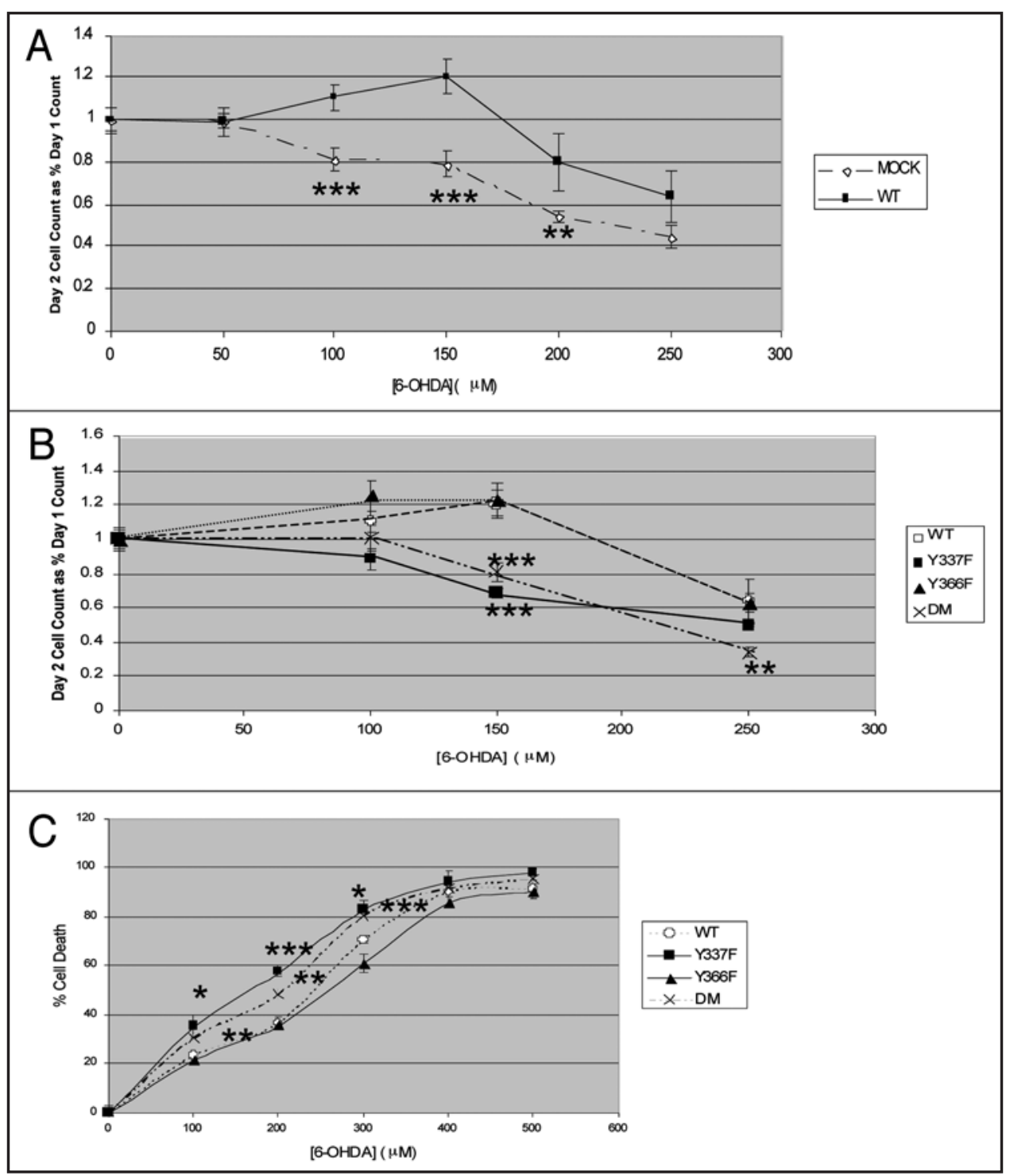

Figure 2. Concentration-response curves (Alamar blue and LDH release determinations of relative cell death; see Experimental Procedure) for $p 75 N T R-$ deficient PC12 cell p75ICD transfectants treated with 6-OHDA. (A) Mock-transfected cells are compared by Alamar blue assay with cells transfected with wildtype (WT) p75ICD; (B) Cells transfected with WT p75ICD are compared by Alamar blue assay with cells transfected, respectively, with each of the mutant $\mathrm{p} 75 \mathrm{ICD}$ constructs. $\mathrm{DM}=\mathrm{Y} 337 \mathrm{~F} / \mathrm{Y} 366 \mathrm{~F}$ p75ICD. Each point represents the mean $\pm \mathrm{SEM}$ of nine independent determinations. (C) Cells transfected with WT $\mathrm{P} 75 \mathrm{ICD}$ are compared by LDH release assay with cells transfected, respectively, with each of the mutant $\mathrm{p} 75 \mathrm{ICD}$ constructs. $D M=Y 337 F / Y 366 F$ p75ICD. Each point represents the mean \pm SEM of 9 independent determinations. For $(A, B$ and $C)$, individual time point results differ from that obtained for WT $p 75$ ICD cells with $p<0.05\left({ }^{*}\right) ; p<0.01\left({ }^{* *}\right)$; or $p<0.001\left(^{* * *}\right)$ as determined by Student's $t$ test.

implicated in p75NTR signaling, and of the activation product of NF- $\kappa$ B, IкB- $\alpha$, were examined in untreated wildtype and mutant p75NTR cells grown in serum- (i.e., NGF-) containing medium. Total ERK1,2 content is similar for all four cell lines. Resting p-ERK1,2 content is highest in wildtype cells and lowest in Y366F cells (Fig. 6). The time course of ERK1,2 activation differs among the transfectants. Mock- and Y337F-transfected cells exhibit biphasic enhancement of p-ERK/Total ERK in the $4 \mathrm{~h}$ following 6-OHDA treatment, with return to baseline levels between phases. By the $4 \mathrm{~h}$ and $1 \mathrm{~h}$ time points, respectively, wildtype and double mutant-transfected cells exhibit a monophasic decrease in p-ERK/ Total ERK, while Y366F cells exhibit a monophasic increase in
p-ERK/Total ERK. Resting p-p38 is lowest in double mutant- and Y366F-transfected cells. Resting $\mathrm{p}-\mathrm{I \kappa B}-\alpha$ content is highest in Y366F cells (Fig. 7).

\section{Discussion}

The neurotrophin receptor, p75NTR, protects PC12 pheochromocytoma cells from oxidant stress. ${ }^{1}$ p75ICD, a cytoplasmic fragment liberated upon ligand binding to the extracellular domain of p75NTR, can substitute for the holoreceptor in this regard. Previous studies have demonstrated that p75ICD phosphorylation plays a role in its activity and in determining which of several p75NTR-triggered signaling pathways is activated. ${ }^{3,4}$ 


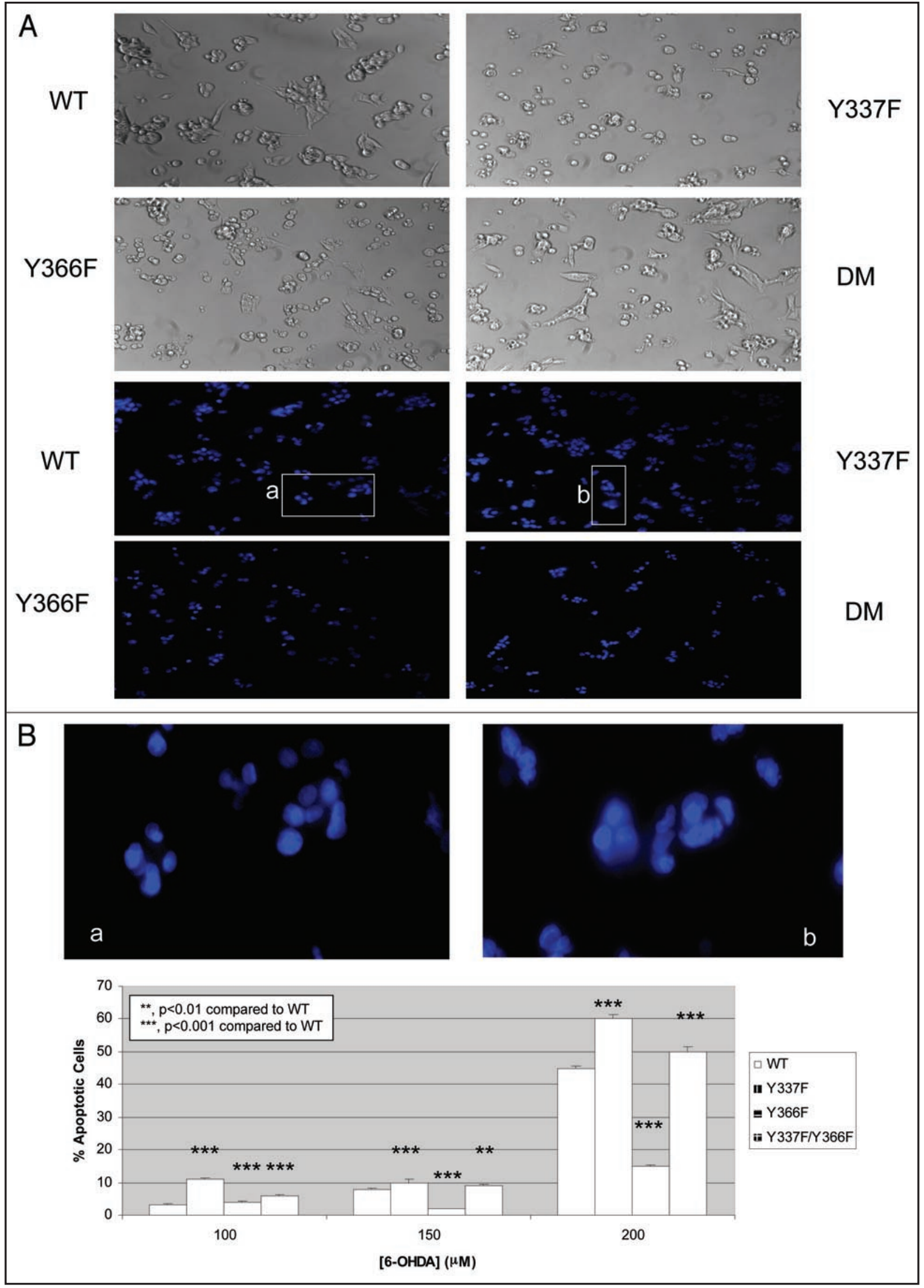


Figure 3. See previous page. Incidence of morphological apoptosis in p75NTR-deficient PC12 cells transfected with wildtype (WT), Y337F, Y366F, or Y337F/Y366F (DM) p75ICD, respectively, treated with 6-OHDA. Representative fields are shown under (A): phase contrast microscopy (10x) and after Hoechst staining followed by fluorescence microscopy (10x), for cells treated with $200 \mu \mathrm{M}$ 6-OHDA. Manual counts of apoptotic and nonapoptotic cells were performed on six fields under fluorescence microscopy for each of the four transfectants. In (B), fields (a and b) from (A) are shown at $200 x$ magnification to demonstrate nuclear condensation and fragmentation and, in the graph, the mean \pm SEM of the manual cell counts is shown ${ }^{* * *} p<0.001$, Student's $t$ test).

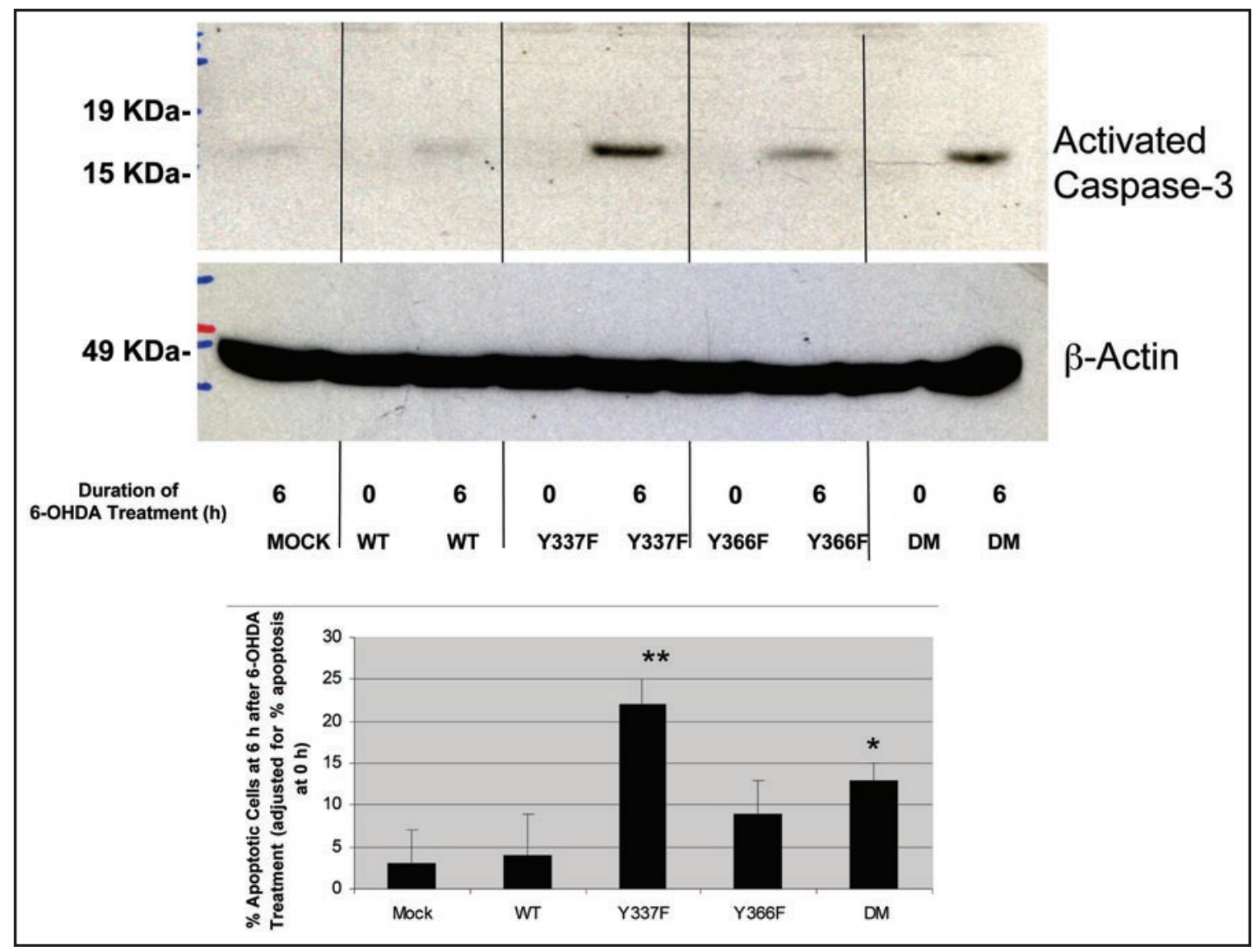

Figure 4. Activated caspase-3 $6 \mathrm{~h}$ after treatment with 6-OHDA $(200 \mu \mathrm{M})$ in p75NTR-deficient PC 12 cells transfected with wiltype (WT), Y337F, Y366F, or Y337F/Y366F (DM) p75ICD, respectively. In the graph, densitometric values for each transfectant before treatment (0 h) are subtracted from values at $6 \mathrm{~h}$ after treatment to obtain the values plotted (mean \pm SEM; ${ }^{*} p<0.01$, ${ }^{*} p<0.05$ relative to WT $p 75 I C D$ transfectant, Student's t-test).

We have used site-directed mutagenesis to generate single and double mutants of p75ICD at Y337 and Y366. The mutants involve substitution of $\mathrm{F}$ for $\mathrm{Y}$ and therefore cannot be phosphorylated at the mutated position. These studies demonstrate that p75NTR-negative PC12 cells transfected with wildtype or Y366F p75ICD are more resistant to oxidative stress than cells that are transfected with Y337F or Y337F/Y366F double mutant p75ICD. As both wildtype and Y366F p75ICD mutant can be phosphorylated on Y337 and both Y337F and Y337F/Y366F cannot, our results suggest that phosphorylation of $\mathrm{Y} 337$ of $\mathrm{p} 75 \mathrm{ICD}$ plays an essential role in the p75ICD-mediated protection of PC12 cells from cell death due to oxidant stress.

Interestingly, as phosphorylation of Y337 is essential for ubiquitination of p75NTR prior to its degradation, ${ }^{4}$ protein levels of the p75ICD Y366F mutant might be expected to be lower than that of the other mutants. On the other hand, in the Y337F mutant, none of Y337 is phosphorylated and thus the p75ICD protein level might be predicted to be highest compared to the other three mutants. However, on average, there were no significant differences in p75ICD content among the cells expressing the four different p75ICD sequences (Fig. 1).

Phosphorylation of p75NTR has been hypothesized to involve transfer of a phosphate from MAPK p38 to p75NTR; there is evidence that p38 can interact directly with the fifth and sixth alpha helices of p75NTR in the C-terminal death domain. ${ }^{8}$ Interestingly, Y366 is located between helix 2 and 3 of p75ICD in the open pocket for $\mathrm{p} 38$. However, our studies demonstrate no correlation between protective activity of phosphorylation site mutants of p75ICD and cellular content of phosphorylated p38 (Fig. 7). Of interest in this regard, members of the MAPK family, including p38 and ERK1,2, have been shown, like p75NTR, to be pro- or anti-apoptotic in neuronal systems and to be critical in oxidant-induced apoptosis in hepatocytes in culture. ${ }^{9,10}$ Furthermore, p38 activation is critical for 6-OHDA-induced 


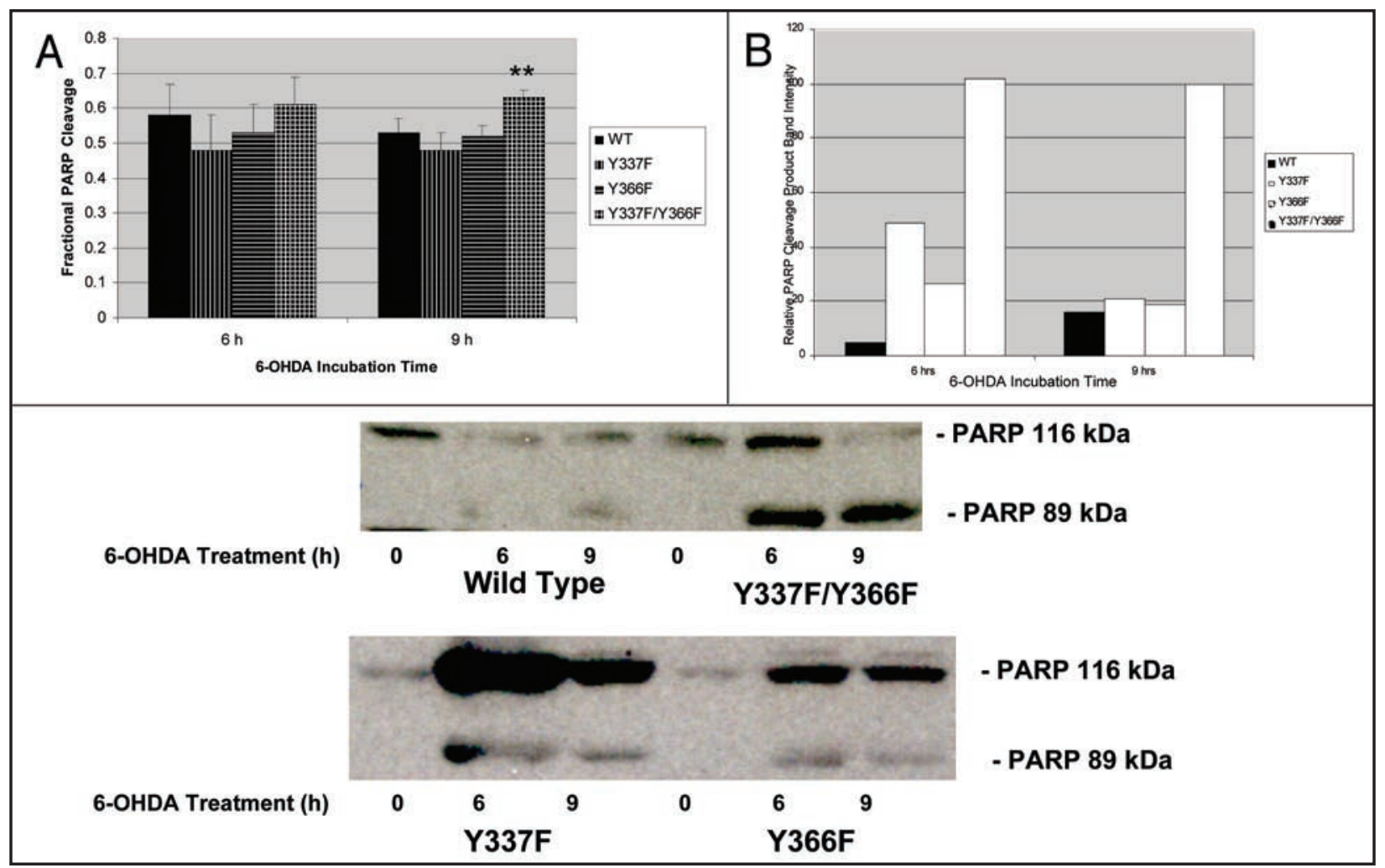

Figure 5. Ratio of PARP cleavage product to total PARP (A) and PARP cleavage product alone (B) determined by measuring the optical density of bands for intact and cleaved PARP on gel electrophoresis of homogenates of 6-OHDA-treated (200 $\mu \mathrm{M})$, p75ICD-transfected, p75NTR-deficient PC12 cells. Results plotted represent the mean \pm SEM of three independent determinations. A single representative set of Western blot results is shown below the graph. ${ }^{* *} p<0.01$, Student's $t$ test)

apoptosis in the CNS-derived tyrosine hydroxylase expressing B65 cell line. ${ }^{11}$ This complex relationship between activation of any of the MAPKs and the life or death of the cell may account for our observation, shown in Figures 6 and 7, that mutants with similar susceptibility to 6-OHDA-induced death have different levels at baseline of activation of the MAPKs p38 and ERK 1,2 relative to wildtype p75ICD cells.

The time course of ERK activation is of particular interest in light of the observation of Luo and DeFranco ${ }^{12}$ that activation of ERK in response to oxidative stress is biphasic and consists of an early, brief, compensatory, protective phase followed by a late, prolonged, cell-injurious phase. Mock- and Y337F-transfected cells demonstrate both of these phases of ERK activation and are most susceptible to 6-OHDA-induced death. Cells transfected with wildtype p75NTR have high levels of activated ERK at baseline and demonstrate a paradoxical decrease in activated ERK at the usual time of the injurious phase of ERK activation. This is perhaps responsible for the protection of these cells from 6-OHDA-induced death. Y366F- and double mutant-transfected cells exhibit ERK activation time courses that do not fit a conventional pattern. Y366F-transfected cells demonstrate early increased ERK activation that increases in magnitude with time, without return to baseline. Given the resistance of these cells to 6-OHDAinduced cell death, it is tempting to speculate that the return to baseline between ERK activation phases is necessary for the transition of p-ERK from protective to injurious. Double mutanttransfected cells demonstrate high baseline levels of ERK activation and early-onset progressively decreasing ERK activation.

Phosphorylation of p75NTR has been shown to enhance NF- $\mathrm{KB}$ activation. ${ }^{8,13,14}$ Our studies demonstrate that activation of NF- $\kappa \mathrm{B}$ is greatest in the Y366F mutant (Fig. 7), implying that phosphorylation of Y366 may inhibit and phosphorylation of Y337 may enhance activation of NF- $\mathrm{KB}$.

Several markers of apoptosis were examined in the present study. Exposure to 6-OHDA results in nuclear margination and fragmentation (Fig. 3A and B) and cleavage of caspase-3 (Fig. 4) and PARP (Fig. 5) in all transfectants. It is interesting that the fractional caspase-3 activation and fractional PARP cleavage for each transfectant do not correlate absolutely and quantitatively with one another or with resistance to oxidant stress. There are two possible, non-mutually exclusive explanations for this. First, it likely that cell death in this model, as in others, ${ }^{15}$ occurs by a combination of apoptosis and necrosis and the relative incidence of these two mechanisms of cell death differ among the transfectants. Second, while useful for the binary decision of whether cells in a given model die by apoptosis or necrosis, Western blotting does not allow for precise kinetic or quantitative comparisons of PARP cleavage from cell line to cell line or tissue to tissue. ${ }^{16}$

The significance of the differing roles in the anti-oxidant effects of p75ICD of phosphorylation of p75ICD at Y337 and Y366, 


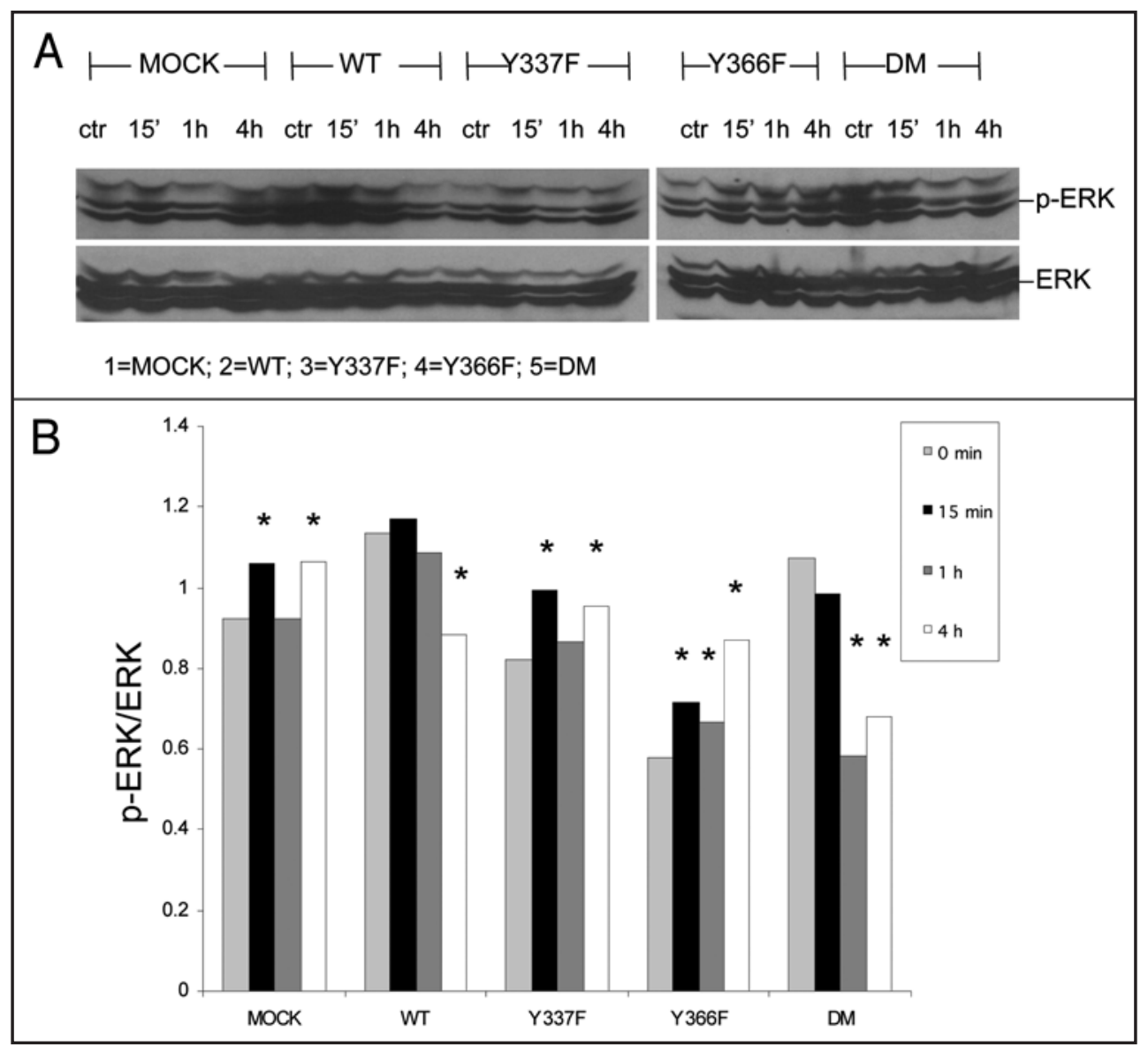

Figure 6. Phosphorylation of ERK1,2 in p75ICD mutants. (A) A representative Western blot of three performed is shown. (B) Densitometric results for $(p-E R K 1+p-E R K 2) /(E R K 1+E R K 2)$ derived from the blot shown in $(A)$, above. *Direction and relative magnitude of difference from $t=0$ min reproduced in all three Western blots.

respectively, for Alzheimer's disease is not clear. Oxidative injury to cholinergic central neurons plays a role in this disorder. In familial Alzheimer's disease, mutation of presenilin, a $\gamma$-secretase for which p75NTR is a substrate, prevents release of p75ICD. However, sporadic Alzheimer's disease, the more common of the two, does not involve mutation of presenilin. Our studies suggest that altered phosphorylation of p75ICD could also result in the enhanced likelihood of apoptotic death of neurons subjected to oxidative stress.

\section{Experimental Procedure}

Chemicals. Ascorbic acid and 6-OHDA were obtained from Sigma Aldrich, Inc. (St. Louis, MO). To prevent extracellular oxidation of 6-OHDA, solutions for use in cell culture experiments were made up in iced saline containing $250 \mu \mathrm{M}$ ascorbic acid with a minimum of agitation immediately before use. Ascorbic acid does not penetrate the cell membrane, and therefore does not alter the intracellular effects of 6-OHDA. Catecholamine transporter-mediated uptake of 6-OHDA by and intracellular generation of reactive oxygen species in PC12 cells have been previously demonstrated. ${ }^{17,18}$

Cell cultures. Polyclonal p75NTR-deficient PC12 cells have been described in our previous publications. ${ }^{5,7}$ Briefly, as a population, native PC12 cells are p75NTR- and TrkA-positive $(-40,000$ p75NTR receptors per cell and -400 TrkA receptors per cell). p75NTR-deficient (0 p75NTR receptors per cell and -400 TrkA receptors per cell) PC12 cells were selected and assayed for receptor content initially and again every several passages as follows. Subclones of PC12 cells were selected on the basis of absent cell-surface p75NTR receptors as determined by FACScan analysis using the monoclonal antibody mAbMC192, a ligand of the extracellular domain of p75NTR. ${ }^{19,20}$ Subclones were then grown and examined by RT-PCR for mRNA for the p75NTR receptor. Studies were performed on pooled subclones, comprising a polyclonal, p75NTR-negative population of PC12 cells.

The p75NTR-deficient PC12 cells were maintained in Dulbecco's modified Eagle's medium (Mediatech, Inc., Manassas, VA) supplemented with $10 \%$ horse serum and $5 \%$ fetal bovine serum (Atlanta Biologicals, Norcross, GA) and $1.1 \%$ penicillin/ streptomycin (Invitrogen, Carlsbad, CA). The cells were fed twice weekly.

Site-directed mutagenesis. Site-directed mutagenesis was carried out following the protocol from Stratagene (La Jolla, CA) for the QuickChange Site-Directed Mutagenesis Kit. The template for PCR was the p75ICD gene sequence cloned into the pcDNA3.1 TOPO V5 His plasmid. ${ }^{1}$ Primers were designed to change $\mathrm{Y}$ to $\mathrm{F}$ 
by changing the $\mathrm{A}$ of the $\mathrm{Y}$ codon (TAC) to $\mathrm{T}$ giving the $\mathrm{F}$ codon (TTC) for both Y337 and Y366. Primers were constructed so that, in each case, the changed nucleotide was the fifth nucleotide from the 5' end. We used forward CTCTTCAGTAGCCTGCCCCTGACC and reverse ACTGAAGAGGTTGCCATCACCCTTG primers in PCR to make Y337F mutants and forward GGTTTCCAGCCTGAACATATAGACTCC and reverse CTGGAAACCCAGCTCGCCTGCCAG primers to make Y366F mutant. PCR was carried out at $72^{\circ} \mathrm{C}$ for extension for 30 cycles of $30 \mathrm{sec}$ each. After Dpn I cleavage, the PCR product was used for transformation and positive colonies were selected and verified by DNA sequencing. The Y337F/Y366F double mutant was made using $\mathrm{Y} 337 \mathrm{~F}$ as a template and the $\mathrm{Y} 366 \mathrm{~F}$ mutant as primers in PCR.

Transfection. After culture in complete medium for several days, the p75NTR-deficient PC12 cells were transfected with plasmids containing either Y337F or Y366F or Y337F/Y366F by electroporation using Amaxa Nucleofector Kit V program U29. After 2 days in regular medium, $500 \mu \mathrm{g} / \mathrm{ml}$ of G418 was added to the medium to select stable positive colonies. Thereafter, the maintenance medium contained $20 \mu \mathrm{g} / \mathrm{ml} \mathrm{G} 418$.

Treatment of cells with 6-OHDA. PC12 cells were seeded on 96-well plates at between 12,000 and 20,000 cells per well and incubated at $37^{\circ} \mathrm{C}$ overnight. The next day, the cells were treated with 6-OHDA in saline containing $250 \mu \mathrm{M}$ ascorbic acid. In the Hoechst staining and PARP cleavage experiments, the final concentration of 6-OHDA was $500 \mu \mathrm{M}$. 6-OHDA remained in the medium until the cells were ready for fixation and staining, or until performance of the Alamar blue assay or SDS PAGE for Western blotting.

Western blotting. For Western blot analysis for p75ICD and intact and cleaved PARP, p75NTR-deficient PC12 cells were lysed in RIPA buffer (10 mM Tris, $\mathrm{pH} 8,150 \mathrm{mM} \mathrm{NaCl}, 0.1 \% \mathrm{NP}-40$, $0.5 \%$ sodium deoxycholate, $0.1 \%$ SDS, $1 \mathrm{mM} \mathrm{PMSF}, 4 \mathrm{mg} / \mathrm{ml}$ aprotinin, $1 \mathrm{mM}$ sodium orthovanadate) for at least $30 \mathrm{~min}$ on ice. Subsequently, the protein concentration was estimated in triplicate samples using the Bio-Rad protein assay (Bio-Rad Laboratories, Hercules, CA, USA) with bovine serum albumin as a standard. Equivalent amounts of protein-containing lysates were loaded onto each lane and electrophoresed in 10\% SDS-polyacrylamide gel, followed by blotting onto a nitrocellulose membrane (Bio-Rad Laboratories). After blotting, non-specific binding was blocked with 5\% non-fat dry milk in PBS for $1 \mathrm{~h}$. The membrane was then incubated for $2 \mathrm{~h}$ at room temperature with anti-p75NTR antibody (Promega, Madison, WI) or overnight at $4^{\circ} \mathrm{C}$ with anti-PARP antibody (Cell Signaling, Danvers, MA), anti-phosphotyrosine antibody (phosphotyrosine clone 2C8 monoclonal antibody; Nano Tools, San Diego, CA), or anti-activated caspase 3 antibody (catalog \#9661; Cell Signaling, Danvers, MA) diluted 1: 1000 into 5\% non-fat dry milk in PBS. The membrane was then washed with PBS, and incubated with secondary horseradish peroxidase-conjugated anti-rabbit IgG antibody or anti-mouse IgG (1:1000; Santa Cruz Biotechnology, Santa Cruz, CA) for $1 \mathrm{~h}$. The membrane was finally washed and developed with Western blotting chemiluminescence luminol reagent (Santa Cruz Biotechnology) following the manufacturer's instructions.

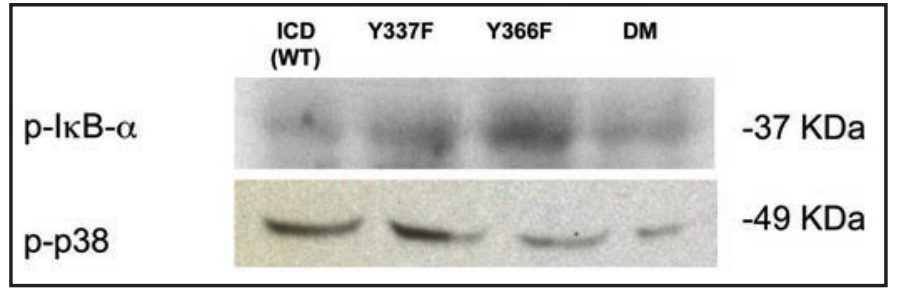

Figure 7. Phosphorylation of the MAPK, p38, and of $1 \kappa B-\alpha$ examined in $\mathrm{p} 75 \mathrm{ICD}$ mutants. A representative Western blot of three performed is shown.

Cell and apoptotic fraction counts. Alamar blue assay. After cells were treated with 6-OHDA for $24 \mathrm{~h}$, the medium was removed and $100 \mu \mathrm{l}$ new medium containing a 1:10 dilution of Alamar blue (Serotec, Oxford, UK) was added to each well and the plate was incubated at $37^{\circ} \mathrm{C}$ for $1 \mathrm{~h}$. Alamar blue fluorescence was determined at an excitation wavelength of $530 \mathrm{~nm}$ and an emission wavelength of $590 \mathrm{~nm}$ using a plate reader.

Hoechst dye staining. PC12 cells were seeded at 20,000 per well onto a 96-well plate at day 0 and treated with 6-OHDA on Day 1. On Day 2, the 6-OHDA-containing medium was removed and the cells were gently washed 3 times with PBS. After adding $50 \mu \mathrm{l}$ Hoechst dye $(20 \mu \mathrm{g} / \mathrm{ml})$ in $4 \%$ paraformaldehyde-PBS to each well, the plate was incubated for $30 \mathrm{~min}$ at room temperature. The Hoechst dye was removed and the wells were washed three times with PBS. The cells were kept in fresh PBS for fluorescent microscopic examination.

LDH cytotoxicity detection assay. PC12 cells were seeded onto 96-well plates on Day 1. On Day 2, when cells were about $70-80 \%$ confluent, they were treated with 6-OHDA at a final concentration of $0,100,200,300,400$ or $500 \mu \mathrm{M}$ for $3 \mathrm{~h}$. Cells were then placed in regular medium. After $24 \mathrm{~h}$, LDH liberated into the overlying medium was quantified using an $\mathrm{LDH}$ Detection Kit (catalogue \#04 744926 001; Roche, Mannheim, Germany) according to the manufacturer's instructions.

Sodium orthovanadate treatment of PC12 cells. Mock- and wild type and mutant p75ICD-transfected PC12 cells were seeded onto $10 \mathrm{~cm}$ culture dishes and grown in DMEM containing 5\% FBS and $10 \%$ horse serum until they attained $80 \%$ confluence. Cells were then washed twice with PBS at room temperature. Equal columes of sodium orthovanadate $(200 \mathrm{mM})$ and $8 \%$ hydrogen peroxide were mixed and immediately pipetted $(10 \mu \mathrm{l})$ into $10 \mathrm{ml}$ warm PBS and added to each dish. The treated cells were incubated at $37^{\circ} \mathrm{C}$ for $5 \mathrm{~min}$. After rinsing with ice cold PBS, the cells were collected using cell scrapers and stored at $-80^{\circ} \mathrm{C}$ until they were ready for Western blot or immunoprecipitation analysis.

Immunoprecipitation. To an aliquot containing $600 \mu \mathrm{g}$ protein and taken from each cell lysate was added RIPA buffer with protease inhibitor and phosphatase inhibitor cocktails (Sigma $8340,2850)$ to make a total volume of $700 \mu \mathrm{l}$. Protein A agarose beads and normal rabbit IgG (Santa Cruz) were then added and the tubes were incubated in a rotor in the cold room for at least $3 \mathrm{~h}$. The beads were then spun down at $4000 \mathrm{~g}$ in the cold room and $10 \mu \mathrm{g}$ anti-human p75 rabbit pAb (Promega G323A) was 
added to the supernatant and incubated overnight in the cold room. The next day, Protein A agarose beads were added again and tubes were incubated in a rotor for at least $3 \mathrm{~h}$ in the cold room. The beads were then spun down and washed with RIPA buffer at least four times and then dissolved into $50 \mu \mathrm{l}$ SDS sample loading buffer for SDS electrophoresis.

\section{Acknowledgements}

The authors thank Dr. Marc Halterman for many helpful discussions and technical advice. The studies were funded through grants from the National Institutes of Health (R01-CA074289 and R01-NS038569) and the William H. Eilinger endowment of the Golisano Children's Hospital at the University of Rochester Medical Center.

References

1. Tyurina YY, Nylander KD, Mirnics ZK, Portugal C, Yan C, Zaccaro C, et al. The intracellular domain of $\mathrm{p} 75 \mathrm{NTR}$ as a determinant of cellular reducing potential and response to oxidant stress. Aging Cell 2005; 4:187-96.

2. Zampieri N, Xu CF, Neubert TA, Chao MV. Cleavage of p75 neurotrophin receptor by alpha-secretase and gamma-secretase requires specific receptor domains. J Biol Chem 2005; 280:14563-71.

3. Blöchl A, Blumenstein L, Ahmadian MR. Inactivation and activation of ras by the neurotrophin receptor p75. Eur J Neurosci 2004; 20:2321-35.

4. Ohrt T, Mancini A, Tamura T, Niedenthal R. c-cbl binds to tyrosine-phosphorylated neurotrophin receptor p75 and induces its ubiquitination. Cell Signal 2004; 16:12918.

5. Mirnics K, Korade Z, Arion D, Lazarov O, Unger T, Macioce M, et al. Presenilin-1dependent transcriptome changes. J Neurosci 2005; 25:1571-8.

6. Korade Z, Mi Z, Portugal C, Schor NF. Expression and p75 neurotrophin receptor dependence of cholesterol synthetic enzymes in adult mouse brain. Neurobiol Aging 2007; 28:1522-31.

7. Yan C, Mirnics ZK, Portugal CF, Liang Y, Nylander KD, Rudzinski M, et al. Cholesterol biosynthesis and the pro-apoptotic effects of the p75 nerve growth factor receptor in PC12 pheochromocytoma cells. Brain Res Mol Brain Res 2005; 139:225-34.

8. Wang JJ, Tasinato A, Ethell DW, Testa MP, Bredesen DE. Phosphorylation of the common neurotrophin receptor $\mathrm{p} 75$ by $\mathrm{p} 38 \beta 2$ kinase affects NF- $\mathrm{KB}$ and AP-1 activities. J Mol Neurosci. 2000; 15:19-29.

9. Miloso M, Scuteri A, Foudah D, Tredici G. MAPKs as mediators of cell fate determination: An approach to neurodegenerative diseases. Curr Med Chem 2008; 15:538-48.

10. Wang X, Li H, Chen Y, Fu J, Ren Y, Dong L, et al. p28GANK knockdown-derived reactive oxygen species induces apoptosis through mitochondrial dysfunction mediated by p38 in HepG2 cells. Int J Oncol 2008; 33:743-50.

11. Kulich SM, Chu CT. Sustained extracellular signal-regulated kinase activation by 6-hydroxydopamine: Implications for parkinson's disease. J Neurochem 2001; 77:105866.

12. Luo Y, DeFranco DB. Opposing roles for ERK1/2 in neuronal oxidative toxicity: Distinct mechanisms of ERK1/2 action at early versus late phases of oxidative stress. J Biol Chem 2006; 281:16436-42.

13. Allen J, Khwaja F, Byers S, Djakiew D. The p75NTR mediates a bifurcated signal transduction cascade through the NFKB and JNK pathways to inhibit cell survival. Exp Cell Res 2005; 304:69-80.

14. Costantini C, Rossi F, Formaggio E, Bernardoni R, Cecconi D, Della-Bianca V. Characterization of the signaling pathway downstream p75 neurotrophin receptor involved in $\beta$-amyloid peptide-dependent cell death. J Mol Neurosci 2005; 25:141-56.

15. Puttonen KA, Lehtonen S, Lampela P, Mannisto PT, Raasmaja A. Different viabilities and toxicity types after 6-OHDA and Ara-C exposure evaluated by four assays in five cell lines. Toxicology in vitro 2008; 22:182-9.

16. Putt KS, Beilman GJ, Hergenrother PJ. Direct quantitation of poly(ADPribose) polymerase (PARP) activity as a means to distinguish necrotic and apoptotic death in cell and tissue samples. Chembiochem 2005; 6:53-5.

17. Eshleman AJ, Neve RL, Janowsky A, Neve KA. Characterization of a recombinant human dopamine transporter in multiple cell lines. J Pharmacol Exp Ther 1995; 274:276-83.

18. Rubenstein R, Price RW. Preservation of catecholamine uptake and release in herpes simplex virus type 1-infected PC12 cells. J Gen Virol 1983; 64:2505-9.

19. Barker PA, Shooter EM. Disruption of NGF binding to the low affinity neurotrophin receptor p75LNTR reduces NGF binding to TrkA on PC12 cells. Neuron 1994; 13:20315.

20. Chandler CE, Parsons LM, Hosang M, Shooter EM. A monoclonal antibody modulates the interaction of nerve growth factor with PC12 cells. J Biol Chem 1984; 259:68829. 


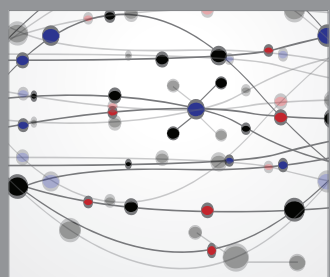

The Scientific World Journal
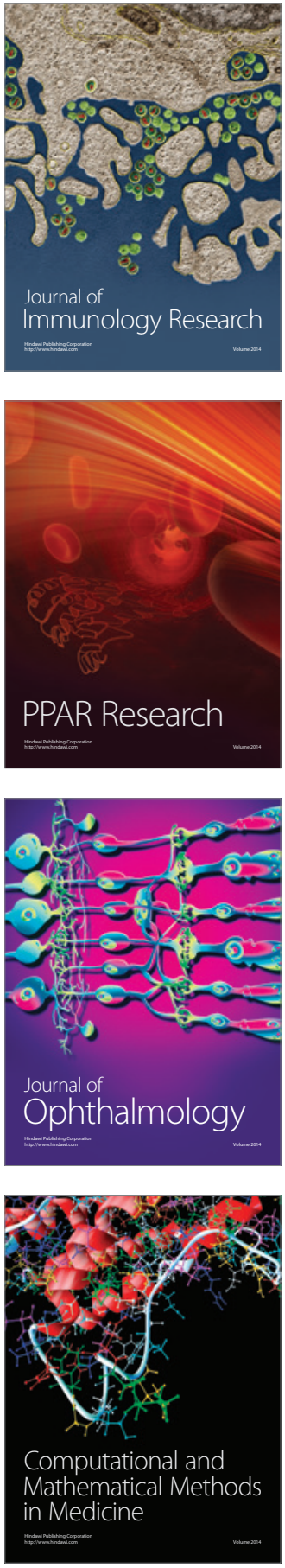

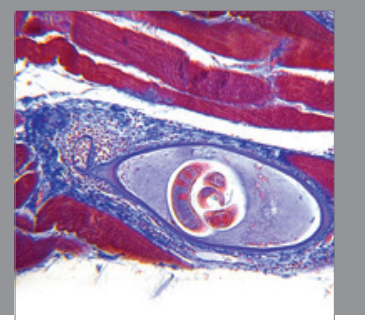

Gastroenterology

Research and Practice
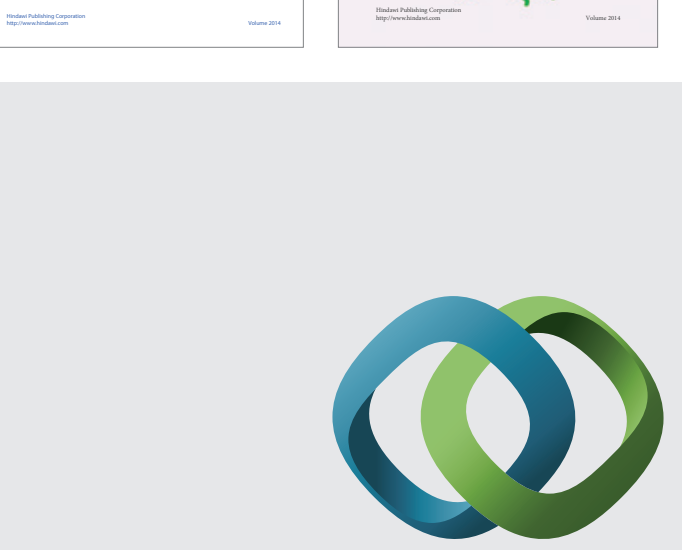

\section{Hindawi}

Submit your manuscripts at

http://www.hindawi.com
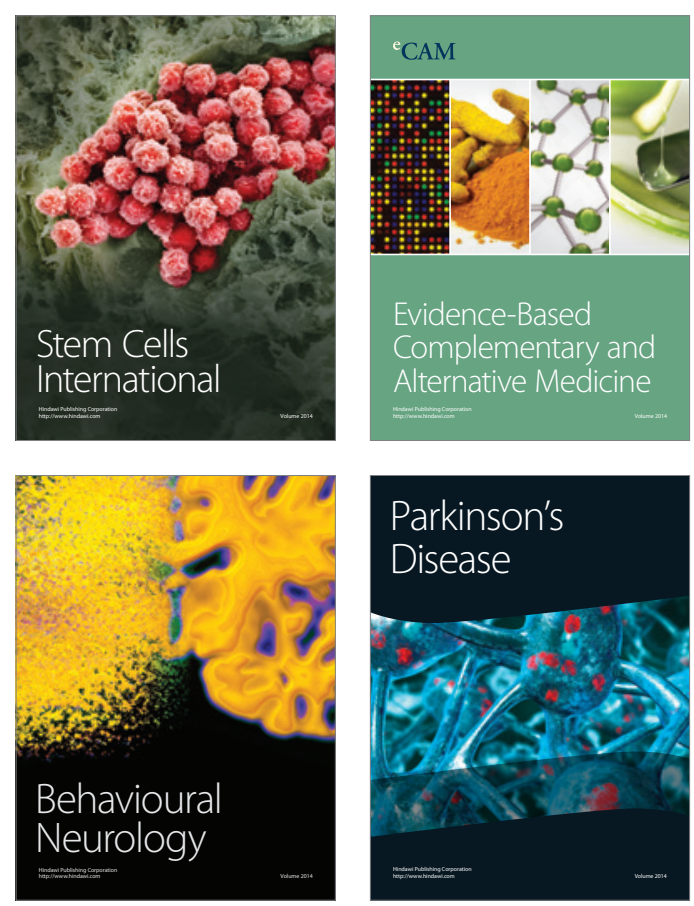

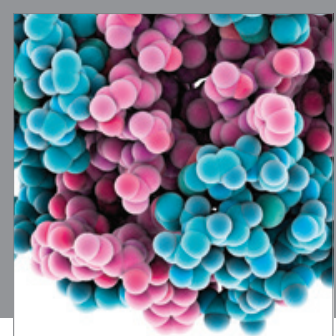

Journal of
Diabetes Research

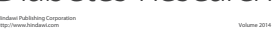

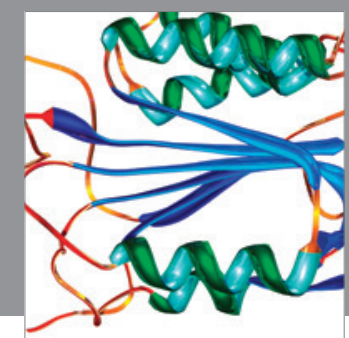

Disease Markers
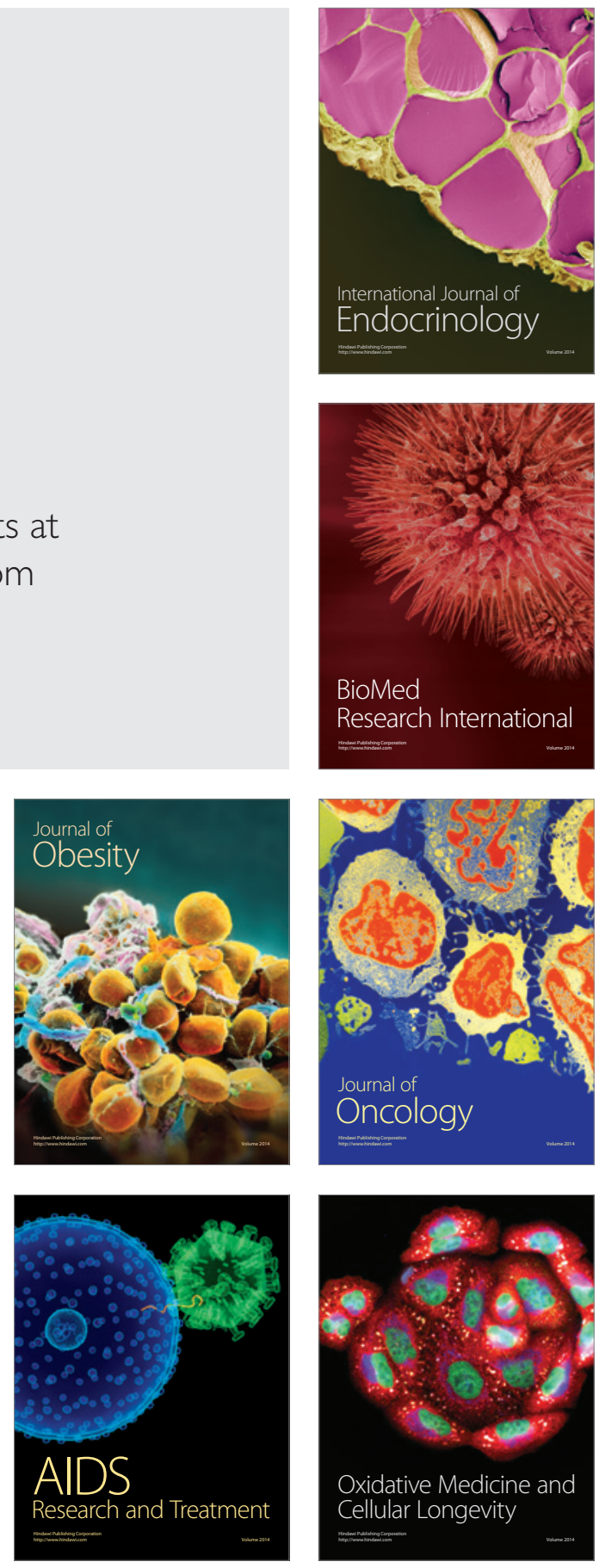